\title{
Event-Triggered Control for Multiagent Systems with the Problem of Packet Losses and Communication Delays When Using the Second-Order Neighbors' Information
}

\author{
Chuan Yan, ${ }^{1}$ Mei Yu, ${ }^{1}$ Guangming Xie, ${ }^{2}$ and Yu Liu ${ }^{3}$ \\ ${ }^{1}$ School of Control and Computer Engineering, North China Electric Power University, Beijing 102206, China \\ ${ }^{2}$ College of Engineering, Peking University, Beijing 100871, China \\ ${ }^{3}$ School of Mathematics and Physics, University of Science and Technology Beijing, Beijing 100083, China \\ Correspondence should be addressed to Mei Yu; yumei@pku.org.cn
}

Received 5 June 2014; Accepted 14 July 2014; Published 17 November 2014

Academic Editor: Abdelghani Bellouquid

Copyright ( 2014 Chuan Yan et al. This is an open access article distributed under the Creative Commons Attribution License, which permits unrestricted use, distribution, and reproduction in any medium, provided the original work is properly cited.

\begin{abstract}
This paper mainly investigates the event-triggered control for discrete-time multiagent systems with the problem of packet losses and communication delays when both the first-order and the second-order neighbors' information are used. Event-triggered control laws are adopted so as to reduce the frequency of individual actuation updating under the sampled-data framework for discrete-time agent dynamics. The communication graph is undirected and the loss of data across each communication link occurs at certain probability, which is governed by a Bernoulli process. It is found that the distributed consensus speeds up by using the secondorder neighbors' information when packet losses and communication delays occur. Numerical examples are given to demonstrate the effectiveness of the proposed methods.
\end{abstract}

\section{Introduction}

In recent years, there has been an increasing research in coordination control of multiagent systems. Information consensus has attracted more and more attention from many engineering application fields, such as formation control, flocking, artificial intelligence, and automatic control [14]. A critical problem in distributed control is to develop distributed protocols under which agents can reach an agreement on a common decision.

Convergence rate is an important index to evaluate the performance of consensus, which has attracted great attention in dealing with this issue [5-8]. In [5], the authors pointed out that the second smallest eigenvalue of its Laplacian matrix was a measure of speed to solve consensus problems. From [6], we know that the convergence speeds up by finding the optimal weight associated with each communication link, where the global structure of the network must be known beforehand. Reference [7] accelerated the convergence rate by using the polynomial filtering algorithms. In
[8], the authors presented randomized gossip algorithm on an arbitrary connected network and showed its performance precisely in terms of the second largest eigenvalue of an appropriate stochastic matrix. The above literatures all tried to seek a suitable topology communication to achieve a fast convergence. However, an excellent protocol can reduce cost, can increase efficiency, and can optimize performance. In practice, it is more useful to design a protocol to obtain a better convergence performance under a given topology. In order to get a better convergence speed without changing the topology and edge weights, the authors in [9] proposed a protocol in an unchanged topology network that each node got its state value updated by using the information of multihop communication for the first time. Then, in [10] the authors discussed that the node in the network topology updated its current state value not only from its immediate neighbors, but also from its second-order neighbors for both the discrete-time case and the continuous-time case. Further, the authors in [11] extended the systems to second-order case and made comparisons between the convergence rate 
of second-order neighbor protocol and the general protocol. Also, the delay margins of general protocol and second-order neighbor protocol were derived.

The literatures mentioned above were mainly considered under the traditional time-triggered control actuation schemes. However, since the microprocessor or controller equipped in each agent may have limited resources or energies in practical applications, it is better for the agents to update their control actuation as little as possible. In $[12,13]$, the authors presented preliminary results of control synthesis for systems with event-based schemes, where the controllers had been designed to ensure stability of the closed-loop systems with respect to measurement errors. Many established results referring to event-triggered schemes are in the framework of continuous-time systems, and some of the results have been extended to discrete-time systems [14]. Reference [15] investigated the consensus problem of discrete-time heterogeneous multiagent systems, where single-integrator and double-integrator agents had been explored under distributed event-based control.

Meanwhile, most of the above works are based on condition of perfect communication link. In reality, the agents exchange data over fading communication channels instead of ideal ones. In fact, in many practical applications, data exchange between sensors is done by wireless communication, which has a possibility of packets losses. Thereby, the packet losses should be taken into consideration. Many related works have been reported. Reference [16] compared the memory and memoryless consensus protocols in the presence of uniform packet losses. In $[17,18]$, the authors discussed the average consensus for first-order agents and analyzed the convergence speed under data losses. Furthermore, [19] showed that packet dropouts could be treated as an absence of a communication link over time. In addition, [20-22] studied stochastic consensus when each edge of the topology was subject to a random process. And then, communication delays are also instable factors which degrade the performance of closed-loop systems in various industry processes. Thereby, the study of time-delay systems has attracted considerable attention over the past years. Reference [23] investigated the problem of accelerating average consensus in undirected and connected network with communication delays for continuous systems. Discrete systems were extended in [24]. Reference [25] investigated the consensus problem of a set of discrete-time heterogeneous multiagent systems with random communication delays. Furthermore, [26] dealt with consensus with random delay and data losses.

Inspired by the above references, we consider eventtriggered control for discrete-time multiagent systems with the problem of packet losses and constant communication delays based on the second-order neighbors' information. We construct a group of agents, which can communicate with their second-order neighbors and each communication link has a probability of failure. We assume that all channels are independent and subject to a distributed random process, which have the same probability of data loss and constant time delay. Each agent is equipped with a sampler and a zero-order hold, which are synchronized. Then, by converting the system to the equivalent error dynamics, stochastic stability of the error dynamic system is studied. Here, a Lyapunov function is constructed and a sufficient condition is established to guarantee the event-based mean square consensus in the form of linear matrix inequality (LMI). We are curious about whether the protocol based on the second-order neighbors' information can accelerate the convergence speed with the problem of packet losses and constant time delay. Finally, a simulation comparison of the convergence rate between the protocol based on the second-order neighbors and the one in general case is shown. Comparisons of the convergence speed between different probabilities of packet losses and constant communication delays are also simulated.

The main contribution of this work is to develop a basic event-triggered control using second-order neighbors' information rather than that of the first one. Moreover, the constant time delay and data losses are explicitly taken into account. It differs from the previous work in such aspects: $[11,13]$ only focus on the effect of the delay, while we focus more on link failure with a probability among agents. Besides, event-triggered control considered in this paper can save energy of agents. Instead of using protocol with firstorder information like in $[19,24]$, we develop the protocol with second-order neighbors' information to accelerate the convergence speed.

The rest of this paper is organized as follows. Section 2 provides some preliminaries on graph theory and gives the designed protocol. Section 3 analyzes the event-based control strategy for multiagent systems without communication delays and gives a sufficient condition on the mean square consensus of the systems. The communication delay of multiagent systems is discussed in Section 4. Section 5 includes some numerical examples. Finally, Section 6 offers the concluding remarks.

Notations. The set of real numbers is denoted by $\mathbb{R}$. For any matrix $Q \in \mathbb{R}^{n \times n}, \operatorname{sym}(Q)=Q+Q^{T}$. The index set $\Lambda_{n}=$ $\{1,2, \ldots n\}$ is a group of consecutive integers from 1 to $n$. The vector $1_{n}=[1,1, \ldots 1]^{T} \in \mathbb{R}^{n}$ has all of its elements equal to 1. The mathematical expectation is denoted by $E\{\cdot\}$ and $P\{\cdot\}$ is the probability operator.

\section{Problem Formation}

2.1. Preliminaries on Graph Theory. In this paper, the interaction among $N$ agents is modeled by an undirected graph $G=$ $(V, \varepsilon, A)$, where $V=\left\{v_{1}, v_{2}, \ldots, v_{n}\right\}$ is the node set. The edge set $\varepsilon \subseteq V \times V$ contains ordered pairs of nodes. The neighbor set of agent $i$ is denoted by $N_{i}$, which includes agents from which agent $i$ receives information. The adjacency matrix $A=\left[w_{i j}\right] \in \mathbb{R}^{n \times n}$ is a nonnegative matrix, where $w_{i j}>0$ if and only if $\left(v_{j}, v_{i}\right) \in \varepsilon$; otherwise, $w_{i j}=0$. We assume that there is no self-loop, so $w_{i i}=0$. The Laplacian matrix $L=\left[l_{i j}\right] \in \mathbb{R}^{n \times n}$ is defined as

$$
l_{i j}=-w_{i j}, \quad \text { if } i \neq j ; \quad l_{i i}=\sum_{k \in N_{i}} w_{i k}
$$


From the above definitions, we know some facts: $A$ and $L$ determine each other uniquely and $L$ has nonnegative eigenvalues. Moreover, $L$ has at least one zero eigenvalue with the associated eigenvector $1_{n}^{T}\left(L 1_{n}=0\right)$; that is, $\operatorname{span}\left\{1_{n}^{T}\right\} \subseteq \operatorname{null}\{L\}$, where null $\{L\}$ is the null space of $L$. For the undirected graph, we further have $L=L^{T}, 1_{n}^{T} L=0$. From [27], it is known that $\operatorname{span}\left\{1_{n}^{T}\right\}=\operatorname{null}\{L\}$ if and only if the undirected graph $G$ is connected.

2.2. Protocols Based on Second-Order Neighbors' Information with Packet Losses. Consider the following first-order dynamics:

$$
\dot{x}_{i}=u_{i}, \quad i \in \Lambda_{n},
$$

where $x_{i}, u_{i} \in \mathbb{R}$ are the state and the input of agent $i$, respectively. With sampling period $T$ and a zero-order hold, the agent dynamics are discretized as

$$
x_{i}(k+1)=x_{i}(k)+T u_{i}(k) \text {. }
$$

The standard consensus protocol that is widely used is proposed as

$$
u_{i}(k)=-r_{c} \sum_{j \in N_{i}} w_{i j}\left(x_{i}(k)-x_{j}(k)\right)
$$

where $r_{c}$ is the control gain.

Consider the protocol based on second-order neighbors' information; if there is no communication constraint taken into account, the following control protocol can be used:

$$
\begin{array}{r}
u_{i}(k)=-r_{c} \sum_{j \in N_{i}} w_{i j}\left[\left(x_{i}(k)-x_{j}(k)\right)\right. \\
\left.+\sum_{h \in N_{j}} w_{j h}\left(x_{i}(k)-x_{h}(k)\right)\right] .
\end{array}
$$

If we consider the packet losses among agents, the following control protocol is designed:

$$
\begin{aligned}
u_{i}(k)=-r_{c}\left\{\sum_{j \in N_{i}} \gamma_{i j}(k) w_{i j}\right. \\
\times\left[\left(x_{i}(k)-x_{j}(k)\right)\right. \\
\left.\left.\quad+\sum_{h \in N_{j}} \gamma_{j h}(k) w_{j h}\left(x_{i}(k)-x_{h}(k)\right)\right]\right\},
\end{aligned}
$$

where $\gamma_{i j}\left(\right.$ or $\left.w_{i j}\right)=1$, if there is no packet loss between agents $i$ and $j ; \gamma_{i j}\left(\right.$ or $\left.w_{i j}\right)=0$, otherwise.

Furthermore, we assume that the occurrence of packet loss is governed by a Bernoulli process with uniform probability $p$ satisfying $0<p<1$; that is,

$$
\begin{array}{r}
P\left\{\gamma_{i j}(k)=1\right\}=p, \quad P\left\{\gamma_{i j}(k)=0\right\}=1-p, \\
\forall i \neq j .
\end{array}
$$

As a result, we have $E\left\{\gamma_{i j}(k)\right\}=p$.

Assumption 1. The undirected topology is coupled; that is, for any pair of agents $i$ and $j$, the communication channels between them exist or vanish simultaneously.

Assumption 1 ensures that the communication topology is always symmetric, so the average of agents' states can be retained during dynamic evolution.

We define two sets of matrices $L_{1}(k)=\left[l_{1 i j}(k)\right] \in \mathbb{R}^{n \times n}$ and $L_{2}(k)=\left[l_{2 i j}(k)\right] \in \mathbb{R}^{n \times n}$ as follows:

$$
\begin{aligned}
& l_{1 i j}(k)=-\gamma_{i j}(k) w_{i j}, \\
& l_{2 i j}(k)=-\sum_{h \in N_{i}, j \in N_{h}} \gamma_{i h}(k) w_{i h} \gamma_{h j}(k) w_{h j}, \quad i \neq j, \\
& l_{1 i i}(k)=\sum_{j \in N_{i}} \gamma_{i j}(k) w_{i j}, \\
& l_{2 i i}(k)=\sum_{h \in N_{i}} \gamma_{i h}(k) w_{i h} \sum_{l \in N_{h}} \gamma_{h l}(k) w_{h l} .
\end{aligned}
$$

Denote the vectors $x(k)$ and $u(k)$ by

$$
\begin{aligned}
& x(k)=\left[x_{1}(k), x_{2}(k), \ldots, x_{n}(k)\right]^{T}, \\
& u(k)=\left[u_{1}(k), u_{2}(k), \ldots, u_{n}(k)\right]^{T} .
\end{aligned}
$$

Then the control protocol can be rewritten as

$$
u(k)=-r_{c} L(k) x(k),
$$

where $L(k)=L_{1}(k)+L_{2}(k)$. So the system dynamics can be written as

$$
\begin{aligned}
x(k+1) & =x(k)-r_{c} T L(k) x(k) \\
& =\left[I-r_{c} T L(k)\right] x(k) .
\end{aligned}
$$

By taking the mathematical expectation of $L_{1}(k)$ and $L_{2}(k)$, we have $E\left\{L_{1}(k)\right\}=p \times L^{(1)}, E\left\{L_{2}(k)\right\}=p^{2} \times L^{(2)}$, where $L^{(1)}$ is the nominal Laplacian matrix of full weights where there is no packet loss and $L^{(2)}$ is the nominal Laplacian matrix of the system which is only based on the second-order neighbors' information with full weights and without packet loss.

Assumption 2. The nominal communication topologies $G^{(1)}$ associated with $L^{(1)}$ and $G^{(2)}$ associated with $L^{(2)}$ are all connected. 
The above assumption is necessary for consensus because if the undirected graph is not connected, then it does not have a spanning tree. From Lemma 1 in [28] and Theorem 5 in [29], we know that there exist two nonempty, disjoint groups of agents that have no communication with each other at any time. In this case, consensus cannot be reached.

\section{No Communication Delays}

In the event-triggered cooperative control strategy, suppose $k_{0}^{i}, k_{1}^{i}, \ldots, k_{l}^{i}, \ldots$ is the sequence of the event times of the agent $i$ which is defined based on the event-triggered condition. We define the state measurement error by

$$
e_{i}(k)=\widehat{x}_{i}(k)-x_{i}(k)
$$

where $\widehat{x}_{i}$ is the latest broadcast state of agent $i$ which is given by $\widehat{x}_{i}(k)=x_{i}\left(k_{l}^{i}\right), k \in\left[k_{l}^{i}, k_{l+1}^{i}\right)$. With $e=\left[e_{1}, \ldots, e_{n}\right]^{T}$, the closed-loop system is described by

$$
x(k+1)=x(k)-r_{c} T L(k)[x(k)+e(k)] .
$$

Furthermore, the average state of the agents

$$
\alpha=\operatorname{Ave}(x(k))=\frac{1}{n} \sum_{i=1}^{n} x_{i}(k)=\frac{1}{n} 1_{n}^{T} x(k)
$$

is invariant. We say the consensus problem is solved, if

$$
\lim \sum_{k=\infty} x_{i}(k)=\alpha, \quad i=1, \ldots, n .
$$

Each agent state can be presented by the form

$$
x(k)=\alpha 1_{n}+\delta(k),
$$

where the variable $\delta=\left[\delta_{1}(k), \delta_{2}(k), \ldots \delta_{n}(k)\right]^{T}$ satisfies

$$
1_{n}^{T} \delta=0
$$

The following error dynamics are obtained:

$$
\begin{aligned}
\delta(k+1) & =\delta(k)-r_{c} T L(k)[\delta(k)+e(k)] \\
& =\left[I-r_{c} T L(k)\right] \delta(k)-r_{c} T L(k) e(k) .
\end{aligned}
$$

Lemma 3. From above, one can get the following conditions:

$$
\begin{aligned}
& E\left(\sum_{j \in N_{i}} \gamma_{i j} w_{i j} \times \sum_{j \in N_{i}} \gamma_{i j} w_{i j}\right) \\
& =p \times \sum_{f=1}^{n} \sum_{g=1}^{n} w_{f g}^{2}+p^{2} \times \sum_{f=1}^{n} \sum_{g=1}^{n} \sum_{m=1}^{n} \sum_{s=1}^{n}\left(w_{f g} w_{m s}\right),
\end{aligned}
$$

$$
\begin{aligned}
& E\left(\sum_{h \in N_{i}} \gamma_{i h} w_{i h} \sum_{l \in N_{h}} \gamma_{h l} w_{h l} \times \sum_{h \in N_{i}} \gamma_{i h} w_{i h} \sum_{l \in N_{h}} \gamma_{h l} w_{h l}\right) \\
& =p^{2} \times \sum_{f=1}^{n} \sum_{g=1}^{n} \sum_{m=1}^{n}\left(w_{f g} w_{g m}\right) \\
& +p^{3} \times \sum_{f=1}^{n} \sum_{g=1}^{n} \sum_{m=1}^{n} \sum_{s=1}^{n}\left(w_{f g}^{2} w_{g m} w_{g s}\right) \\
& +p^{3} \times \sum_{f=1}^{n} \sum_{g=1}^{n} \sum_{m=1}^{n} \sum_{s=1}^{n}\left(w_{f g} w_{g m}^{2} w_{s g}\right) \\
& +p^{4} \times \sum_{f=1}^{n} \sum_{g=1}^{n} \sum_{m=1}^{n} \sum_{s=1}^{n} \sum_{u=1}^{n} \sum_{v=1}^{n}\left(w_{f g} w_{g m} w_{m u} w_{u v}\right) .
\end{aligned}
$$

Then for an undirected graph, given the Laplacian matrices $L_{1}(k), L_{2}(k)$ and a symmetric matrix $Q,\left(L^{(1)}+L^{(2)}\right) Q\left(L^{(1)}+\right.$ $\left.L^{(2)}\right)$ can be calculated as follows:

$$
\left(L^{(1)}+L^{(2)}\right) Q\left(L^{(1)}+L^{(2)}\right)=\left[\begin{array}{ccc}
A_{11} & \cdots & A_{1 n} \\
\vdots & \ddots & \vdots \\
A_{n 1} & \cdots & A_{n n}
\end{array}\right]_{n \times n},
$$

where

$$
\begin{aligned}
& L^{(1)}+L^{(2)} \\
& =\left[\begin{array}{ccc}
\sum_{j \in N_{i}} w_{1 j}+\sum_{h \in N_{i}} w_{1 h} \sum_{l \in N_{h}} w_{h l} & \cdots & -w_{1 n}-w_{1 n} \sum_{l \in N_{h}} w_{n l} \\
\vdots & \ddots & \vdots \\
-w_{n 1}-w_{n 1} \sum_{l \in N_{h}} w_{1 l} & \cdots & \sum_{j \in N_{i}} w_{n j}+\sum_{h \in N_{i}} w_{n h} \sum_{l \in N_{h}} w_{h l}
\end{array}\right]_{n \times n},
\end{aligned}
$$$$
A_{11}=\left[\left(\sum_{j \in N_{i}} w_{1 j}+\sum_{h \in N_{i}} w_{1 h} \sum_{l \in N_{h}} w_{h l}\right) q_{11}\right.
$$$$
\left.+\cdots-\left(w_{1 n}+w_{1 n} \sum_{l \in N_{h}} w_{h l}\right) q_{n 1}\right]
$$$$
\times\left[\left(\sum_{j \in N_{i}} w_{1 j}+\sum_{h \in N_{i}} w_{1 h} \sum_{l \in N_{h}} w_{h l}\right)\right]
$$$$
+\cdots+\left[\left(\sum_{j \in N_{i}} w_{1 j}+\sum_{h \in N_{i}} w_{1 h} \sum_{l \in N_{h}} w_{h l}\right) q_{1 n}\right.
$$

$$
\left.+\cdots-\left(w_{1 n}+w_{1 n} \sum_{l \in N_{h}} w_{h l}\right) q_{n n}\right]
$$


Abstract and Applied Analysis

5

$$
\begin{aligned}
& \times\left[-w_{n 1}-w_{n 1} \sum_{l \in N_{h}} w_{1 l}\right], \\
& A_{1 n}=\left[\left(\sum_{j \in N_{i}} w_{1 j}+\sum_{h \in N_{i}} w_{1 h} \sum_{l \in N_{h}} w_{h l}\right) q_{11}\right. \\
& \left.+\cdots-\left(w_{1 n}+w_{1 n} \sum_{l \in N_{h}} w_{h l}\right) q_{n 1}\right] \\
& \times\left[-w_{1 n}-w_{1 n} \sum_{l \in N_{h}} w_{n l}\right] \\
& +\cdots+\left[\left(\sum_{j \in N_{i}} w_{1 j}+\sum_{h \in N_{i}} w_{1 h} \sum_{l \in N_{h}} w_{h l}\right) q_{1 n}\right. \\
& \left.+\cdots-\left(w_{1 n}+w_{1 n} \sum_{l \in N_{h}} w_{h l}\right) q_{n n}\right] \\
& \times\left[\left(\sum_{j \in N_{i}} w_{n j}+\sum_{h \in N_{i}} w_{n h} \sum_{l \in N_{h}} w_{h l}\right)\right], \\
& A_{n 1}=\left[\left(\sum_{j \in N_{i}} w_{1 j}+\sum_{h \in N_{i}} w_{1 h} \sum_{l \in N_{h}} w_{h l}\right) q_{11}\right. \\
& \left.+\cdots-\left(w_{1 n}+w_{1 n} \sum_{l \in N_{h}} w_{h l}\right) q_{n 1}\right] \\
& \times\left[\left(\sum_{j \in N_{i}} w_{1 j}+\sum_{h \in N_{i}} w_{1 h} \sum_{l \in N_{h}} w_{h l}\right)\right] \\
& +\cdots+\left[\left(\sum_{j \in N_{i}} w_{1 j}+\sum_{h \in N_{i}} w_{1 h} \sum_{l \in N_{h}} w_{h l}\right) q_{1 n}\right. \\
& \left.+\cdots-\left(w_{1 n}+w_{1 n} \sum_{l \in N_{h}} w_{h l}\right) q_{n n}\right] \\
& \times\left[-w_{n 1}-w_{n 1} \sum_{l \in N_{h}} w_{1 l}\right], \\
& A_{n n}=\left[\left(\sum_{j \in N_{i}} w_{1 j}+\sum_{h \in N_{i}} w_{1 h} \sum_{l \in N_{h}} w_{h l}\right) q_{11}\right. \\
& \left.+\cdots-\left(w_{1 n}+w_{1 n} \sum_{l \in N_{h}} w_{h l}\right) q_{n 1}\right]
\end{aligned}
$$

$$
\begin{gathered}
\times\left[-w_{1 n}-w_{1 n} \sum_{l \in N_{h}} w_{n l}\right] \\
+\cdots+\left[\left(\sum_{j \in N_{i}} w_{1 j}+\sum_{h \in N_{i}} w_{1 h} \sum_{l \in N_{h}} w_{h l}\right) q_{1 n}\right. \\
\left.+\cdots-\left(w_{1 n}+w_{1 n} \sum_{l \in N_{h}} w_{h l}\right) q_{n n}\right] \\
\times\left[\left(\sum_{j \in N_{i}} w_{n j}+\sum_{h \in N_{i}} w_{n h} \sum_{l \in N_{h}} w_{h l}\right)\right] .
\end{gathered}
$$

Then combining with $L_{1}(k)$ and $L_{2}(k), \quad E\left\{\left(L_{1}(k)+\right.\right.$ $\left.\left.L_{2}(k)\right) Q\left(L_{1}(k)+L_{2}(k)\right)\right\}$ can be achieved. The following theorem gives a sufficient condition on the event-based consensus of the system.

Theorem 4. Given the scalar $r_{c}$, if there exists a matrix $Q>0$, such that the following LMI holds

$$
F_{1}=\left[\begin{array}{cc}
-A_{1}+\sigma I & C_{1} \\
B_{1} & -D_{1}-I
\end{array}\right]<0,
$$

where $\sigma$ is a positive scalar which satisfies $0<\sigma<1$, the mean square consensus of the system (3) is achieved asymptotically under the triggered condition given by

$$
\left\|e_{i}(k)\right\|_{2}^{2} \leq \sigma\left\|\delta_{i}(k)\right\|_{2}^{2},
$$

where

$$
\begin{aligned}
\bar{P} & =E(L(k) Q L(k)), \\
D_{1} & =E\left(-r_{c}^{2} T^{2} L(k) Q L(k)\right)=-r_{c}^{2} T^{2} \bar{P}, \\
B_{1} & =E\left(r_{c} T\left\{L(k) Q\left(r_{c} T L(k)-I\right)\right\}\right) \\
& =-r_{c} T p\left(L^{(1)} Q+p L^{(2)} Q\right)+r_{c}^{2} T^{2} \bar{P}, \\
C_{1} & =E\left(r_{c} T\left(\left(r_{c} T L(k)-I\right) Q L(k)\right)\right) \\
& =-r_{c} T p\left(Q L^{(1)}+p Q L^{(2)}\right)+r_{c}^{2} T^{2} \bar{P}, \\
A_{1} & =E\left\{r_{c} T\left(L(k) Q+Q L(k)-r_{c} T L(k) Q L(k)\right)\right\} \\
& =r_{c} T p\left[L^{(1)} Q+Q L^{(1)}+p \times\left(L^{(2)} Q+Q L^{(2)}\right)\right]-r_{c}^{2} T^{2} \bar{P} .
\end{aligned}
$$


Proof. Construct the candidate Lyapunov function as $V(k)=$ $\delta^{T}(k) Q \delta(k)$. We have

$$
\begin{aligned}
E\{\Delta & V(k)\} \\
\leq & E\{V(k+1)-V(k)\}+\sigma \delta^{T}(k) \delta(k)-e^{T}(k) e(k) \\
\leq & E\left\{\delta^{T}(k+1) Q \delta(k+1)-\delta^{T}(k) Q \delta(k)\right\} \\
& +\sigma \delta^{T}(k) \delta(k)-e^{T}(k) e(k) \\
\leq & E\left\{\left[\left[I-r_{c} T L(k)\right] \delta(k)-r_{c} T L(k) e(k)\right]^{T}\right. \\
& \times Q\left[\left[I-r_{c} T L(k)\right] \delta(k)-r_{c} T L(k) e(k)\right] \\
& \left.\quad-\delta^{T}(k) Q \delta(k)\right\} \\
\leq & -\delta^{T}(k) \delta_{1} \delta(k)+e^{T}(k) B_{1} \delta(k) \\
& +\delta^{T}(k) C_{1} e(k)-e^{T}(k) D_{1} e(k) \\
& +\sigma \delta^{T}(k) \delta(k)-e^{T}(k) e(k) \\
\leq & \xi^{T}(k) F_{1} \xi(k),
\end{aligned}
$$

where $\xi(k)=\left[\begin{array}{ll}\delta^{T}(k) & e^{T}(k)\end{array}\right]^{T}$.

Thus, the subsequent task is to show that the error dynamic system is stable in the mean square sense. Let the largest eigenvalue of $F_{1}$ be $\lambda_{\text {max }}$; then

$$
\begin{aligned}
E\{V(k+1)-V(k)\} & \leq \xi^{T}(k) F_{1} \xi(k) \\
& \leq \lambda_{\max }\|\xi(k)\|^{2}
\end{aligned}
$$

Summing (26) from $k=0$, it can be obtained that

$$
\begin{aligned}
E\{V(k+1)-V(0)\} & \leq \lambda_{\max } \sum_{m=0}^{k} E\left\{\|\xi(m)\|^{2}\right\}, \\
\sum_{m=0}^{k} E\left\{\|\xi(m)\|^{2}\right\} & \leq-\frac{1}{\lambda_{\max }} E\{V(0)-V(k+1)\} .
\end{aligned}
$$

Let $k \rightarrow \infty$; the following inequality can be derived:

$$
\begin{aligned}
\sum_{m=0}^{k} E\left\{\|\xi(m)\|^{2}\right\} & \leq-\frac{1}{\lambda_{\max }} E\{V(0)-V(\infty)\} \\
& \leq-\frac{1}{\lambda_{\max }} E\{V(0)\} .
\end{aligned}
$$

Considering that $\|\xi(m)\|^{2} \geq\|\delta(m)\|^{2}, m=0,1,2, \ldots$, therefore,

$$
\begin{aligned}
\sum_{m=0}^{k} E\left\{\|\delta(m)\|^{2}\right\} & \leq \sum_{m=0}^{k} E\left\{\|\xi(m)\|^{2}\right\} \\
& \leq-\frac{1}{\lambda_{\max }} E\{V(0)\}<\infty
\end{aligned}
$$

Therefore, it can be concluded that $\lim _{m \rightarrow \infty} E\left\{\|\delta(m)\|^{2}\right\}=$ 0 , and thus the error dynamic system in (13) is mean square stable. So consensus is achieved in the mean square sense.

\section{With Communication Delays}

Based on second-order neighbors' information with communication delays and packet losses, the control protocol can be described as

$$
\begin{aligned}
& u_{i}(k) \\
& =-r_{c}\left\{\sum_{j \in N_{i}} \gamma_{i j}(k) w_{i j}\right. \\
& \times\left[\begin{array}{c}
\left(x_{i}(k)-x_{j}(k)\right) \\
+\sum_{h \in N_{j}} \gamma_{j h}(k) w_{j h} \\
\times\left(x_{i}(k-\tau)-x_{h}(k-\tau)\right)
\end{array}\right\},
\end{aligned}
$$

where $\tau$ is a constant delay. Then, using protocol (30) and measurement error (12), the system has the following form:

$$
\begin{aligned}
\delta(k+1)= & \delta(k)-r_{c} T L_{1}(k)[\delta(k)+e(k)] \\
& -r_{c} T L_{2}(k)[\delta(k-\tau)+e(k-\tau)] .
\end{aligned}
$$

Theorem 5. Given the scalar $r_{c}$, if there exist matrices $Q>0$, $S>0$, and $R>0$, such that the following LMI holds 
$F_{2}$

$=\left[\begin{array}{cccc}-r_{c} T p\left(L^{(1)} Q+Q L^{(1)}\right)+r_{c}^{2} T^{2} \bar{P}_{11}+S+\sigma I & -2 r_{c} T p Q L^{(1)}+2 r_{c}^{2} T^{2} \bar{P}_{12} & -2 r_{c} T p Q L^{(1)}+2 r_{c}^{2} T^{2} \bar{P}_{11} & -2 r_{c} T p Q L^{(1)}+2 r_{c}^{2} T^{2} \bar{P}_{12} \\ * & r_{c}^{2} T^{2} \bar{P}_{22}-S & 2 r_{c}^{2} T^{2} \bar{P}_{21} & 2 r_{c}^{2} T^{2} \bar{P}_{22} \\ * & * & r_{c}^{2} T^{2} \bar{P}_{11}+R-I & r_{c}^{2} T^{2} \bar{P}_{12} \\ * & * & * & r_{c}^{2} T^{2} \bar{P}_{22}-R\end{array}\right]$

$<0$,

the mean square consensus of the system (31) is achieved asymptotically under the event-triggered condition given by

$$
f_{i}=\left\|e_{i}(k)\right\|_{2}^{2}-\sigma\left\|\delta_{i}(k)\right\|_{2}^{2},
$$

where $\sigma$ is a given positive scalar which satisfies $0<$ $\sigma<1, \bar{P}_{11}=E\left(L_{1}(k) Q L_{1}(k)\right), \bar{P}_{12}=$ $E\left(L_{1}(k) Q L_{2}(k)\right), \bar{P}_{21}=E\left(L_{2}(k) Q L_{1}(k)\right)$, and $\bar{P}_{22}=$ $E\left(L_{2}(k) Q L_{2}(k)\right)$.

Proof. Construct the candidate Lyapunov function as

$$
\begin{aligned}
V(k)= & \delta^{T}(k) Q \delta(k) \\
& +\sum_{i=k-\tau}^{k-1} \delta^{T}(i) S \delta(i)+\sum_{i=k-\tau}^{k-1} e^{T}(i) \operatorname{Re}(i) .
\end{aligned}
$$

Combining the event condition in (33), we have

$$
\begin{aligned}
E\{\Delta V(k)\} \leq & E\{V(k+1)-V(k)\} \\
& +\sigma \delta^{T}(k) \delta(k)-e^{T}(k) e(k) \\
\leq & \epsilon^{T}(k) F_{2} \epsilon(k),
\end{aligned}
$$

where $\epsilon(k)=\left[\begin{array}{llll}\delta^{T}(k) & \delta^{T}(k-\tau) & e^{T}(k) & e^{T}(k-\tau)\end{array}\right]^{T}$.

Thus, the subsequent task is to show that the error dynamic system is stable in the mean square sense. Let the largest eigenvalue of $F_{2}$ be $\lambda_{\max }(r)<0$; then

$$
\begin{aligned}
E\{V(k+1)-V(k)\} & \leq \epsilon^{T}(k) F_{2} \epsilon(k) \\
& \leq \lambda_{\max }\|\epsilon(k)\|^{2} .
\end{aligned}
$$

Summing (36) from $k=0$, it can be obtained that

$$
\begin{aligned}
E\{V(k+1)-V(0)\} & \leq \lambda_{\max } \sum_{m=0}^{k} E\left\{\|\epsilon(m)\|^{2}\right\}, \\
\sum_{m=0}^{k} E\left\{\|\epsilon(m)\|^{2}\right\} & \leq-\frac{1}{\lambda_{\max }} E\{V(0)-V(k+1)\} .
\end{aligned}
$$

Let $k \rightarrow \infty$; the following inequality can be derived:

$$
\begin{aligned}
\sum_{m=0}^{k} E\left\{\|\epsilon(m)\|^{2}\right\} & \leq-\frac{1}{\lambda_{\max }} E\{V(0)-V(\infty)\} \\
& \leq-\frac{1}{\lambda_{\max }} E\{V(0)\} .
\end{aligned}
$$

Considering that $\|\epsilon(m)\|^{2} \geq\|\delta(m)\|^{2}, m=0,1,2, \ldots$, therefore,

$$
\begin{aligned}
\sum_{m=0}^{k} E\left\{\|\delta(m)\|^{2}\right\} & \leq \sum_{m=0}^{k} E\left\{\|\epsilon(m)\|^{2}\right\} \\
& \leq-\frac{1}{\lambda_{\max }} E\{V(0)\}<\infty
\end{aligned}
$$

Therefore, it can be concluded that $\lim _{m \rightarrow \infty} E\left\{\|\delta(m)\|^{2}\right\}=$ 0 , and thus the error dynamic system in (31) is mean square stable. So consensus is achieved in the mean square sense.

\section{Simulations and Analyses}

To illustrate fast convergence rate of the protocol for the consensus of the system (2) based on second-order neighbors' information, numerical examples are provided for secondorder multiagent systems. The nominal interaction topology and the topology based on second-order neighbors' information among five agents are shown in Figure 1.

The weights are set to unity for simplicity here. The corresponding Laplacian matrices $L^{(1)}$ and $L^{(2)}$ are given as follows:

$$
L^{(1)}=\left[\begin{array}{ccccc}
2 & -1 & 0 & 0 & -1 \\
-1 & 2 & -1 & 0 & 0 \\
0 & -1 & 2 & -1 & 0 \\
0 & 0 & -1 & 2 & -1 \\
-1 & 0 & 0 & -1 & 2
\end{array}\right]
$$

$$
L^{(2)}=\left[\begin{array}{ccccc}
2 & 0 & -1 & -1 & 0 \\
0 & 2 & 0 & -1 & -1 \\
-1 & 0 & 2 & 0 & -1 \\
-1 & -1 & 0 & 2 & 0 \\
0 & -1 & -1 & 0 & 2
\end{array}\right] \text {. }
$$

The loss of data across each communication link occurs at certain probability; then, we choose the probability of successfully receiving information as $p=0.9$. The time history of Bernoulli variable $\gamma_{a b}(k)$ is shown in Figure 2.

We choose the sampling period $T=0.05 \mathrm{~s}$, control gain $r_{c}=0.5$, and the triggered parameters $\eta_{\max }=0.001$, $\sigma=0.005$ and the initial condition is set to be $x(0)=$ $[1,2,3,4,5]$. It will be shown that the agents' states finally converge to the average value $\alpha=\operatorname{Ave}(x(0))=(1+2+3+$ 


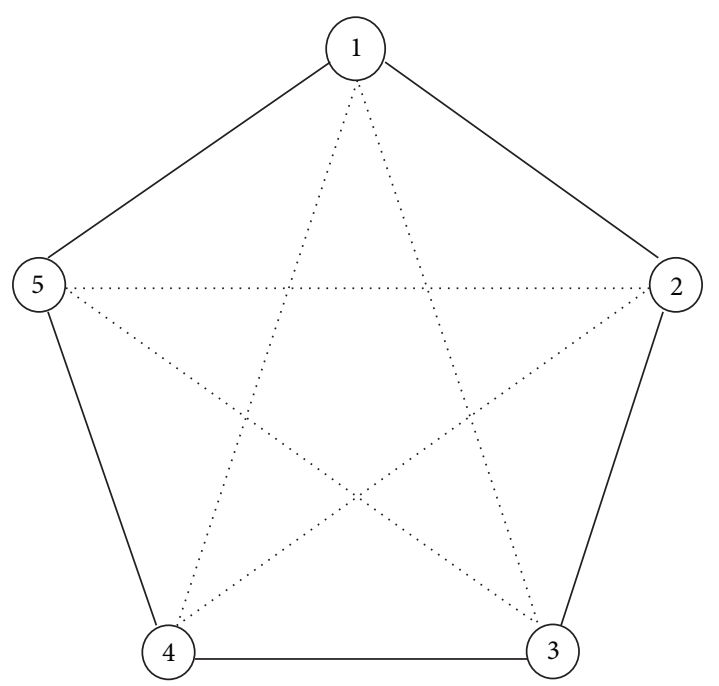

FIGURE 1: Nominal communication topology based on the secondorder neighbors' information.

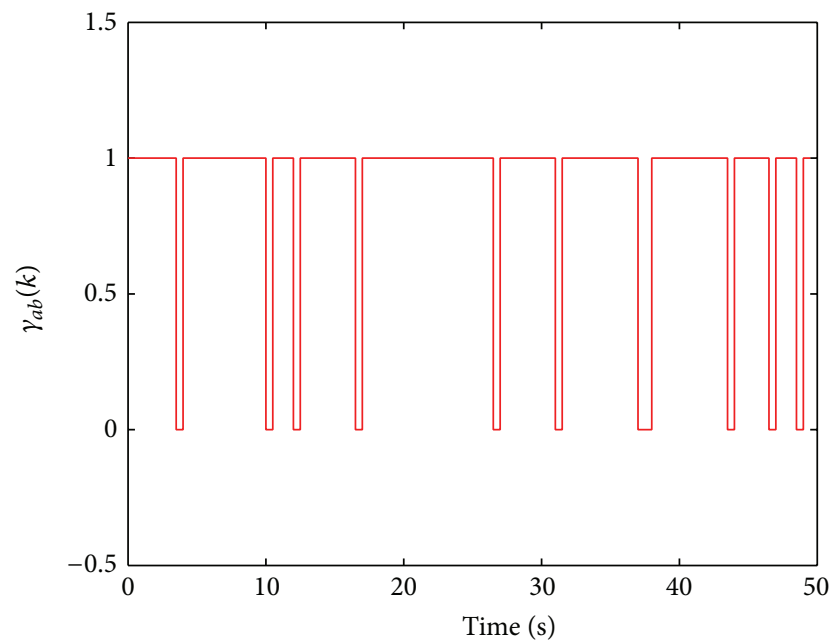

FIgURE 2: Time history of Bernoulli variable $\gamma_{a b}(k)$.
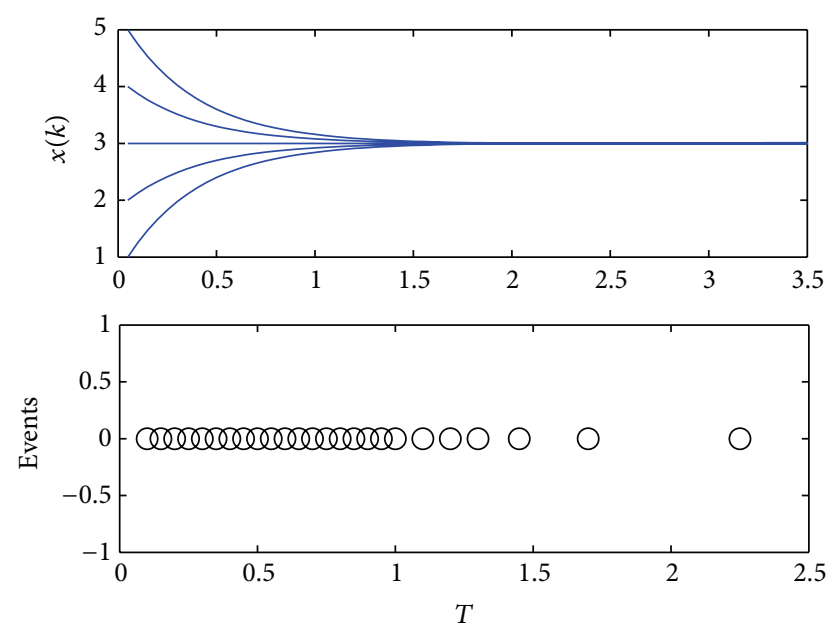

Figure 3: Protocol (6) for $p=0.9$.
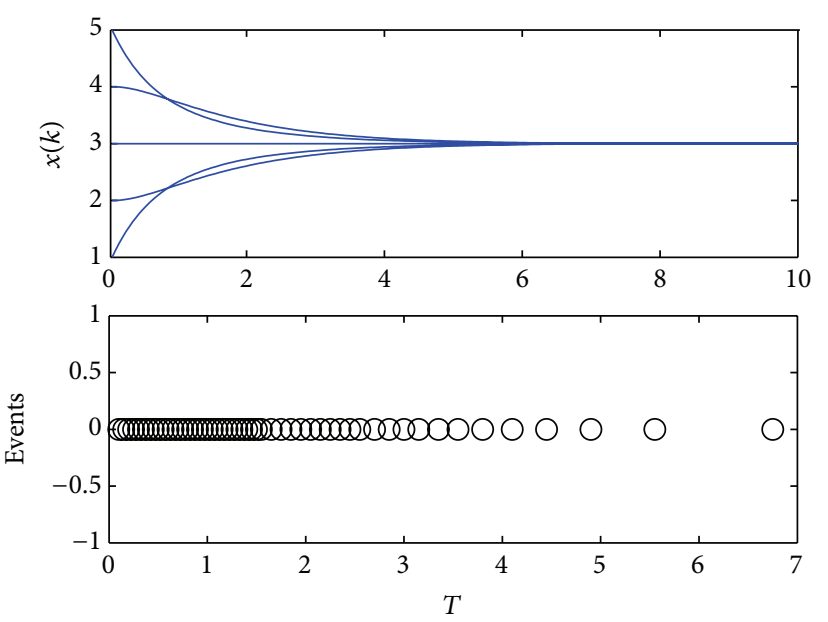

FIGURE 4: Protocol (4) for $p=0.9$.
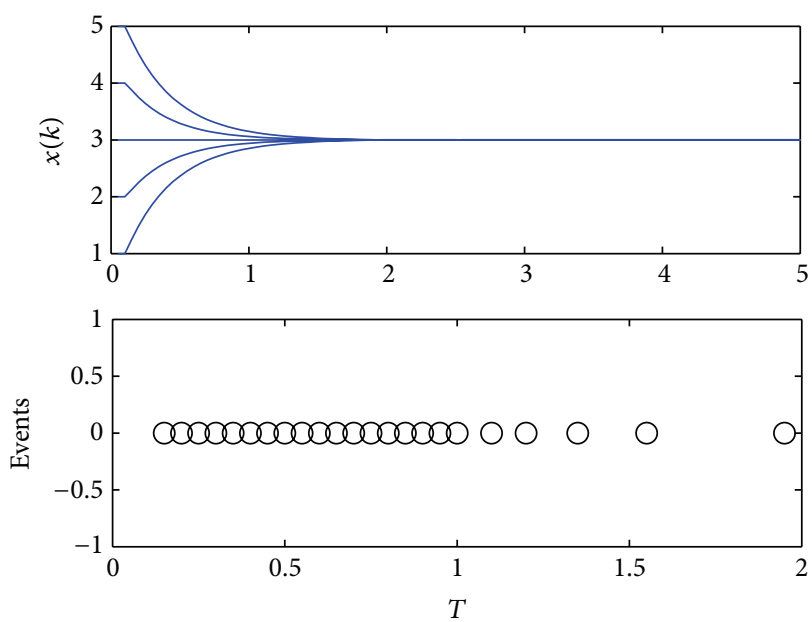

Figure 5: Protocol (26) for $p=0.9$ and $\tau=0.025 \mathrm{~s}$.

$4+5) \div 5=3$. Then, by solving the LMIs in Theorems 4 and 5 the results show that they are feasible. Thus consensus will be achieved for the system with and without time delays. Figure 3 shows the state trajectories and event times of the agents under protocol (6) when $p=0.9$. Figure 4 shows the state trajectories and event times of the agents under protocol (4) when $p=0.9$, from which we know that in this case the system converges faster under protocol (6) than under protocol (4). Similarly, Figures 5 and 6 show the simulation results of protocol (26) with $\tau=0.025 \mathrm{~s}$ for $p=0.9$ and $p=0.5$, respectively, from which we know that the system converges faster under lower probability of packet losses. Figures 5 and 7 show the states evolution and event times under protocol (26) with $p=0.9$ for $\tau=0.025 \mathrm{~s}$ and $\tau=0.1 \mathrm{~s}$, respectively. It is clear that the delay margin of Figure 5 is smaller than that of Figure 7; the system runs faster and the number of actuator updates reduces greatly for smaller time delay. 

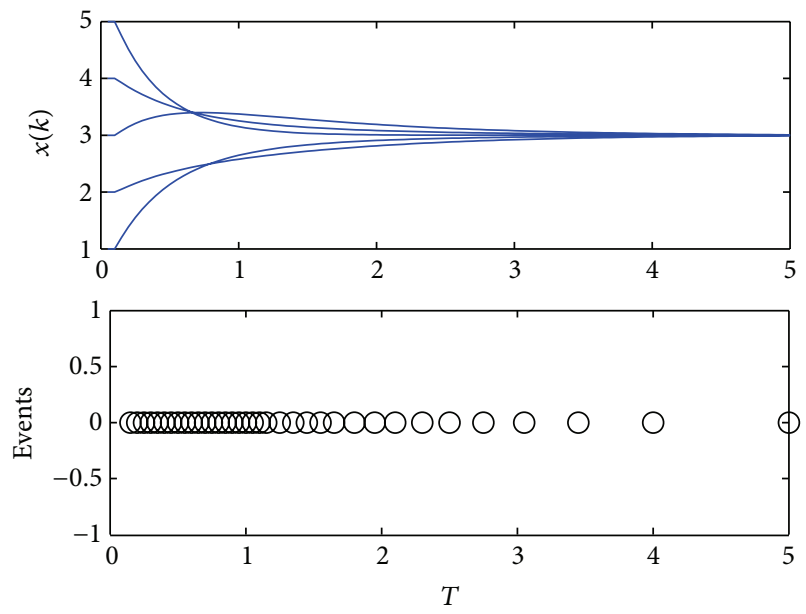

Figure 6: Protocol (26) for $p=0.5$ and $\tau=0.025 \mathrm{~s}$.
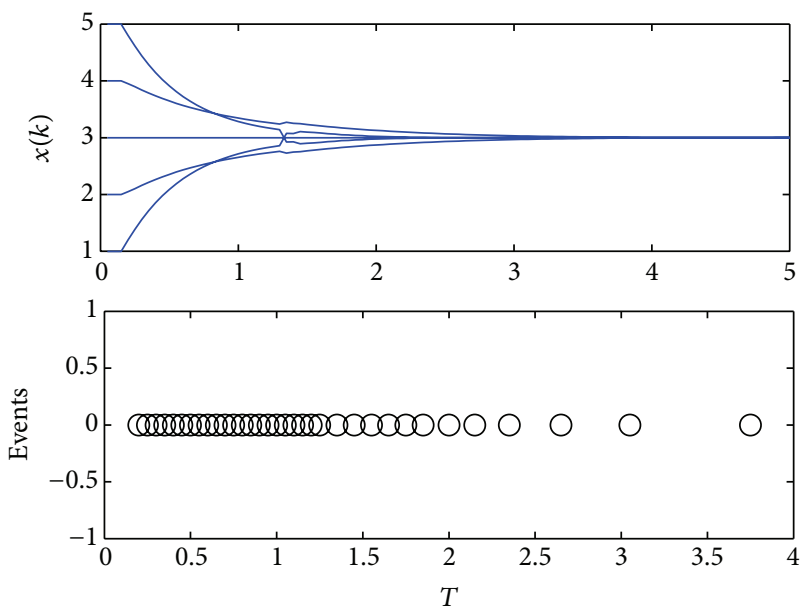

Figure 7: Protocol (26) for $p=0.9$ and $\tau=0.1 \mathrm{~s}$.

\section{Conclusion}

In this paper, we have investigated the event-based consensus for the second-order multiagent systems with the problem of packet losses and communication delays when second-order neighbors' information is used. The convergence rates of general protocol and second-order neighbor protocol have been compared and it is concluded that second-order neighbor protocol speeds up the consensus rate. What is more, we can see the influence of packet losses and communication delays on the convergence of the system. Future work will include the agent dynamics with time-varying delay.

\section{Conflict of Interests}

The authors declare that there is no conflict of interests regarding the publication of this paper.

\section{Acknowledgments}

This work is supported by Beijing Municipal Natural Science Foundation (4122075), the National Natural Science Foundation of China under grants (no. 61004031, no. 61174096, and no. 61104141), and the Fundamental Research Funds for the Central Universities and Program for New Century Excellent Talents in University.

\section{References}

[1] F. Xiao, L. Wang, J. Chen, and Y. Gao, "Finite-time formation control for multi-agent systems," Automatica, vol. 45, no. 11, pp. 2605-2611, 2009.

[2] F. Xiao, L. Wang, and T. Chen, "Connectivity preservation for multi-agent rendezvous with link failure," Automatica, vol. 48, no. 1, pp. 25-35, 2012.

[3] H. Michael, Z. Chen, X. Wang, and J. Lam, "Semi-global observer-based leader-following consensus with input saturation," IEEE Transactions on Industrial Electronics, vol. 61, no. 6, pp. 2842-2850, 2014.

[4] L. Wang and F. Xiao, "Finite-time consensus problems for networks of dynamic agents," IEEE Transactions on Automatic Control, vol. 55, no. 4, pp. 950-955, 2010.

[5] R. Olfati-Saber and R. M. Murray, "Consensus problems in networks of agents with switching topology and time-delays," IEEE Transactions on Automatic Control, vol. 49, no. 9, pp. 15201533, 2004.

[6] L. Xiao and S. Boyd, "Fast linear iterations for distributed averaging," Systems and Control Letters, vol. 53, no. 1, pp. 65-78, 2004.

[7] E. Kokiopoulou and P. Frossard, "Polynomial filtering for fast convergence in distributed consensus," IEEE Transactions on Signal Processing, vol. 57, no. 1, pp. 342-354, 2009.

[8] S. Boyd, A. Ghosh, B. Prabhakar, and D. Shah, "Randomized gossip algorithms," IEEE Transactions on Information Theory, vol. 52, no. 6, pp. 2508-2530, 2006.

[9] Z. P. Jin and R. Murray, "Multi-hop relay protocols for fast consensus seeking," in Proceedings of the 45th IEEE Conference on Decision and Control (CDC '06), pp. 1001-1006, December 2006

[10] D. Yuan, S. Xu, H. Zhao, and Y. Chu, "Accelerating distributed average consensus by exploring the information of secondorder neighbors," Physics Letters A, vol. 374, no. 24, pp. 2438 2445, 2010.

[11] H. Pan, X. Nian, and L. Guo, "Second-order consensus in multiagent systems based on second-order neighbours' information," International Journal of Systems Science, vol. 45, no. 5, pp. 902914, 2014.

[12] X. Wang and M. D. Lemmon, "Decentralized event-triggered broadcasts over networked control systems," in Hybrid Systems: Computation and Control, vol. 4981 of Lecture Notes in Computer Science, pp. 674-677, Springer, Berlin, Germany, 2008.

[13] X. Wang and M. Lemmon, "On event design in event-triggered feedback systems," Automatica, vol. 47, no. 10, pp. 2319-2322, 2011.

[14] A. Eqtami, D. V. Dimarogonas, and K. J. Kyriakopoulos, "Eventtriggered control for discrete-time systems," in Proceedings of the American Control Conference (ACC '10), pp. 4719-4724, July 2010. 
[15] X. Yin, X. Yue, and S. Hu, "Distributed event-triggered control of discrete-time heterogeneous multi-agent systems," Journal of the Franklin Institute, vol. 350, no. 3, pp. 651-669, 2013.

[16] S. Wang, C. An, X. Sun, and X. Du, "Average consensus over communication channels with uniform packet losses," in Proceeding of the Chinese Control and Decision Conference (CCDC '10), pp. 114-119, Xuzhou, China, May 2010.

[17] F. Fagnani and S. Zampieri, "Randomized consensus algorithms over large scale networks," IEEE Journal on Selected Areas in Communications, vol. 26, no. 4, pp. 634-649, 2008.

[18] F. Fagnani and S. Zampieri, "Average consensus with packet drop communication," SIAM Journal on Control and Optimization, vol. 48, no. 1, pp. 102-133, 2009.

[19] J. Wu and Y. Shi, "Average consensus in multi-agent systems with time-varying delays and packet losses," in Proceedings of the IEEE American Control Conference (ACC '12), pp.1579-1584, June 2012.

[20] Y. Hatano and M. Mesbahi, "Agreement over random networks," IEEE Transactions on Automatic Control, vol. 50, no. 11, pp. 1867-1872, 2005.

[21] C. W. Wu, "Synchronization and convergence of linear dynamics in random directed networks," IEEE Transactions on Automatic Control, vol. 51, no. 7, pp. 1207-1210, 2006.

[22] M. Porfiri and D. J. Stilwell, "Consensus seeking over random weighted directed graphs," IEEE Transactions on Automatic Control, vol. 52, no. 9, pp. 1767-1773, 2007.

[23] L. Rong, S. Xu, B. Zhang, and Y. Zou, "Accelerating average consensus by using the information of second-order neighbours with communication delays," International Journal of Systems Science, vol. 44, no. 6, pp. 1181-1188, 2013.

[24] V. Kapila and W. M. Haddad, "Memoryless $H_{\infty}$ controllers for discrete-time systems with time delay," Automatica, vol. 34, no. 9, pp. 1141-1144, 1998.

[25] X. Yin and D. Yue, "Event-triggered tracking control for heterogeneous multi-agent systems with Markov communication delays," Journal of the Franklin Institute, vol. 350, no. 5, pp. 13121334, 2013.

[26] Y. Zhang and Y. Tian, "Consensus of data-sampled multi-agent systems with random communication delay and packet loss," IEEE Transactions on Automatic Control, vol. 55, no. 4, pp. 939943, 2010.

[27] W. Ren and R. W. Beard, "Consensus seeking in multiagent systems under dynamically changing interaction topologies," IEEE Transactions on Automatic Control, vol. 50, no. 5, pp. 655661, 2005.

[28] Z. Lin, B. Francis, and M. Maggiore, "Necessary and sufficient graphical conditions for formation control of unicycles," IEEE Transactions on Automatic Control, vol. 50, no. 1, pp. 121-127, 2005.

[29] L. Moreau, "Stability of multiagent systems with time-dependent communication links," IEEE Transactions on Automatic Control, vol. 50, no. 2, pp. 169-182, 2005. 


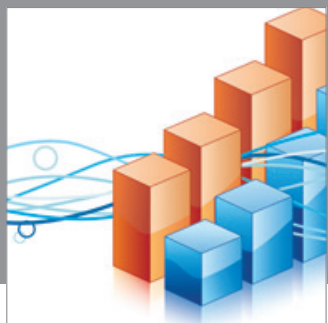

Advances in

Operations Research

mansans

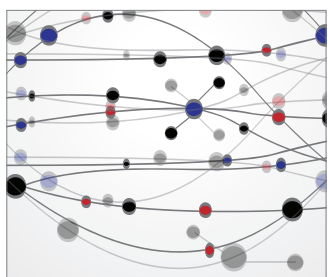

The Scientific World Journal
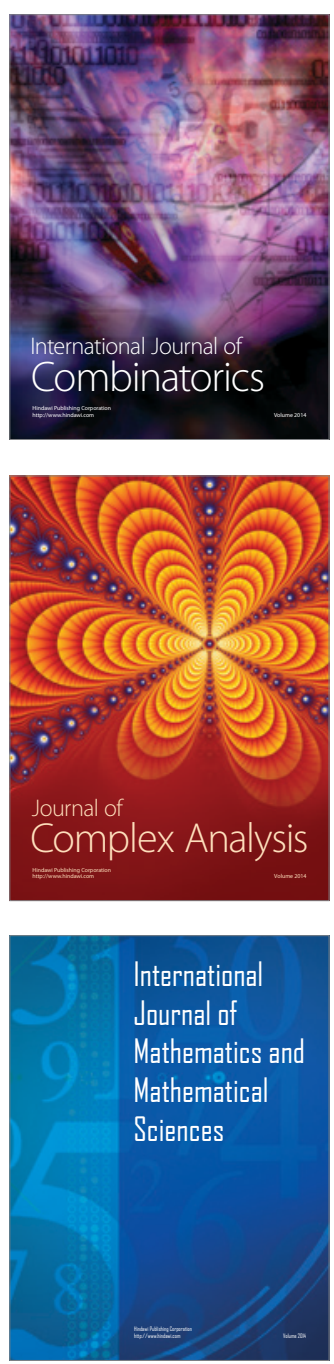
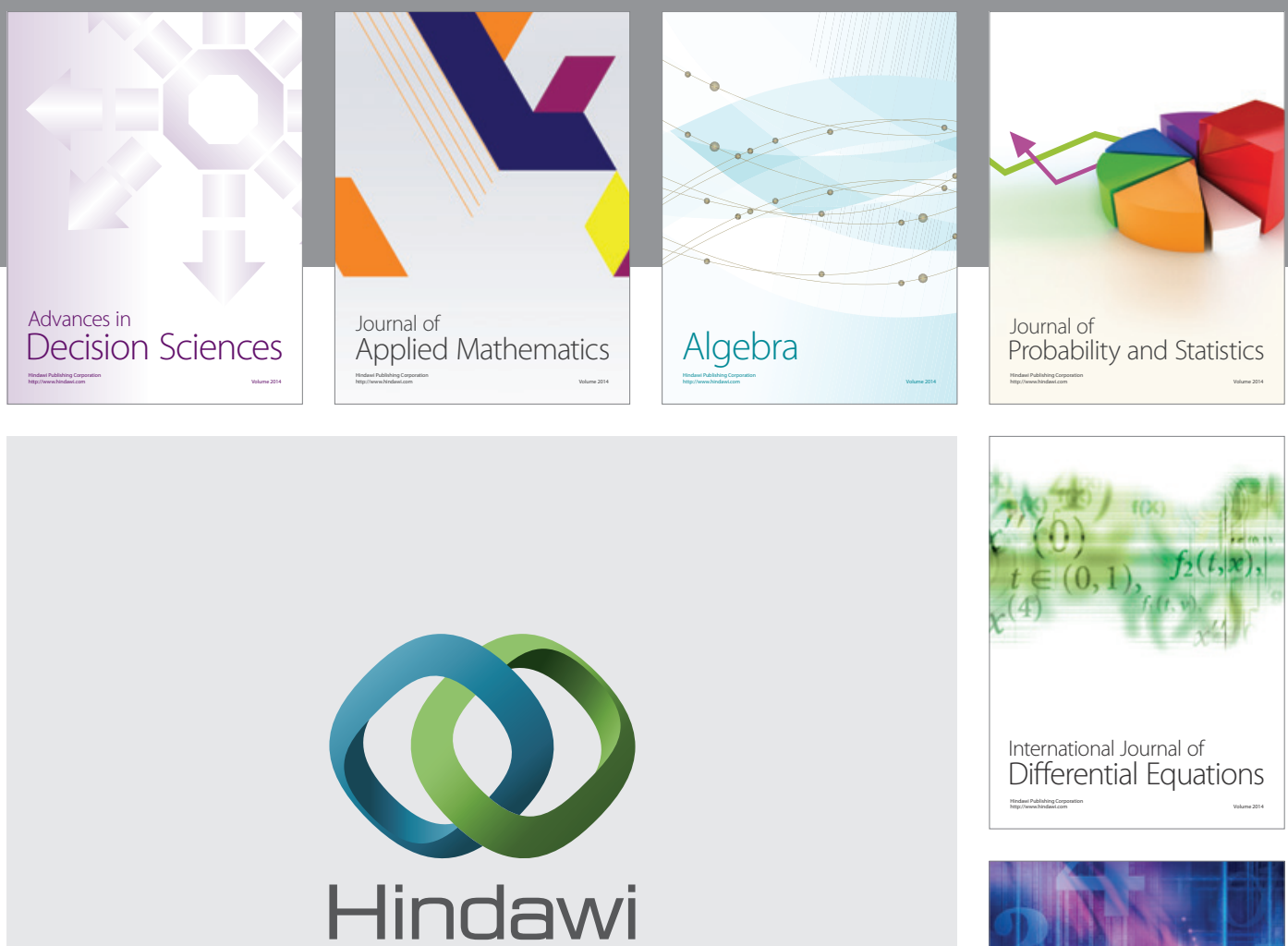

Submit your manuscripts at http://www.hindawi.com
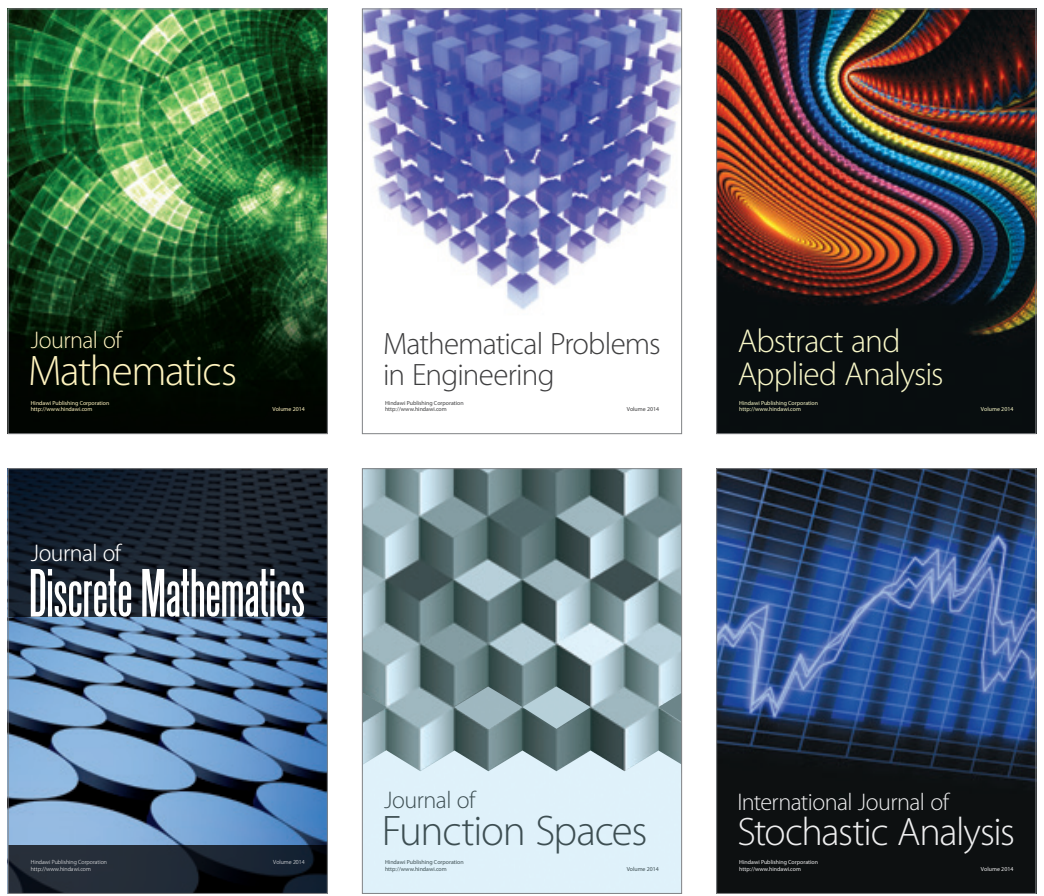

Journal of

Function Spaces

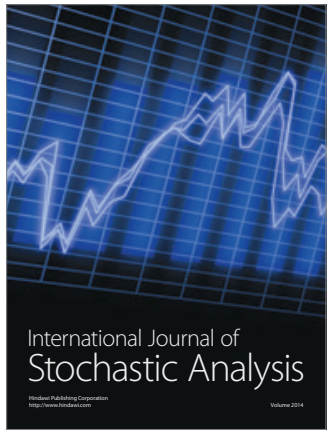

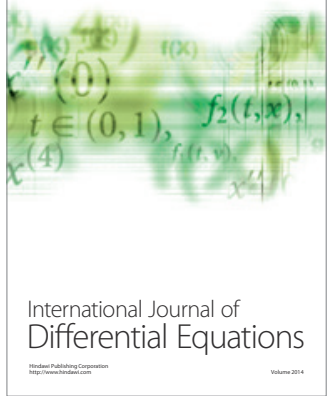
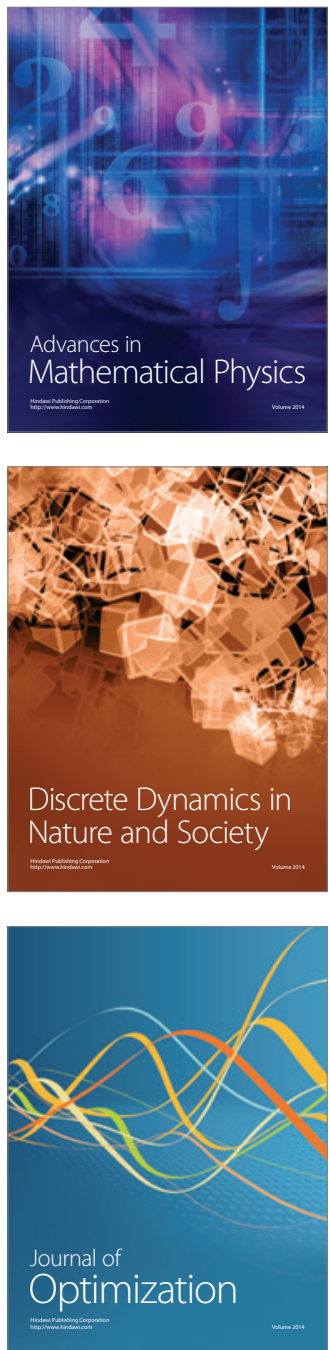